\title{
Search for spontaneous edge currents and vortex imaging in $\mathrm{Sr}_{2} \mathrm{RuO}_{4}$ mesostructures
}

\author{
P. J. Curran, ${ }^{1}$ S. J. Bending, ${ }^{1}$ W. M. Desoky, ${ }^{1,2}$ A. S. Gibbs, ${ }^{3}$ S. L. Lee, ${ }^{3}$ and A. P. Mackenzie ${ }^{3,4}$ \\ ${ }^{1}$ Department of Physics, University of Bath, Claverton Down, Bath BA2 7AY, United Kingdom \\ ${ }^{2}$ Department of Physics, Zagazig University, Ash Sharqiyah, Egypt \\ ${ }^{3}$ University of St. Andrews, School of Physics \& Astronomy, Scottish Universities Physics Alliance, St. Andrews KY16 9SS, Fife, Scotland \\ ${ }^{4}$ Max-Planck Institute for Chemical Physics of Solids, D-01187 Dresden, Germany
}

(Received 7 January 2014; revised manuscript received 6 March 2014; published 9 April 2014)

\begin{abstract}
Scanning Hall probe microscopy has been used to search for spontaneous fields at the well-defined edges of large mesoscopic disks, etched into the $a b$ surface of very high quality single crystal $\mathrm{Sr}_{2} \mathrm{RuO}_{4}$. Such fields are predicted to exist at locations of broken translational symmetry as a consequence of the proposed two-component spin-triplet chiral order parameter $\hat{d}=\Delta_{0}\left(k_{x} \pm i k_{y}\right) \hat{z}$. We find no evidence for such fields and impose an upper limit of $\pm 2.5 \mathrm{mG}$ on their magnitude. We do, however, observe an abrupt apparent loss of strong bulk pinning and a change in the screening behavior above $H \sim 25$ Oe. At high fields $(H>25 \mathrm{Oe})$ pronounced magnetic screening by the disks is very well described by a model containing only strong edge currents, and bulk critical currents do not appear to play a significant role. Our results are discussed in terms of relevant theoretical predictions.
\end{abstract}

\section{INTRODUCTION}

Unconventional superconductors are of great scientific interest because they potentially play host to Cooper pair binding mechanisms that are different from the electron-phonon interaction of conventional superconductivity [1]. The different spatial distributions of higher angular momentum pairing mean that the electrons of Cooper pairs sit in very different Coulomb potentials, and are likely to be stabilized by different pairing mechanisms. Therefore, in order to inform theoretical developments, the pairing symmetry in a material must first be unambiguously established, and in this regard the scientific debate on $\mathrm{Sr}_{2} \mathrm{RuO}_{4}$ is ongoing [2,3].

Early Knight-shift measurements strongly suggested that the superconductivity in $\mathrm{Sr}_{2} \mathrm{RuO}_{4}$ is spin triplet [4], with $p$ wave being favored due to energetic considerations [5]. Evidence for broken time reversal symmetry came from muon spin rotation $(\mu \mathrm{SR})[6]$ and polar-Kerr measurements [7] which, along with considerations of the crystal structure of $\mathrm{Sr}_{2} \mathrm{RuO}_{4}$ [8], uniquely identified a two-component chiral $p$-wave order parameter $\left[\hat{d}=\Delta_{0}\left(k_{x} \pm i k_{y}\right) \hat{z}\right.$ in $d$-vector notation] as the most likely candidate. However, additional experimental evidence for this is inconclusive [3,9]. Much interest surrounds an apparent discrepancy between theory and experiment regarding spontaneous currents (fields) that are expected to arise as a direct consequence of such a pairing symmetry wherever translational symmetry is broken [2]. These "smoking gun" signals, predicted at sample edges and domain walls formed between regions of opposite chirality $[10,11]$, have been proposed as the source of the internal fields detected by early $\mu \mathrm{SR}$ measurements [6], but have so far evaded detection by real-space scanning probe measurements despite predictions that they should be readily detectable [12,13].

Recent scanning Hall probe microscopy (SHPM) measurements on $\mathrm{Sr}_{2} \mathrm{RuO}_{4}$ illustrated that striking differences in behavior can be observed between single crystals from different growth batches, even if they are grown under the same nominal conditions [14]. Such sensitivity to crystal quality highlights the importance of extending the search for chiral signals to crystals from different batches with the highest possible degree of order. Scanning superconducting quantum interference device (SQUID) measurements were able to image over the as-grown edge of a single crystal [13,15], but as yet no measurements over a controlled, well-defined microstructured boundary have taken place. Here we describe a series of measurements of mesoscopic disks, etched into the surface of a very high quality sample. The disk edges provide well-defined locations where translational symmetry is broken and so would be expected to host observable chiral edge fields. Theoretical investigations of mesoscopic (sample dimensions $\sim \lambda, \xi$ ) chiral $p$-wave superconductors also predict the emergence of several new magnetic phenomena such as chirality-dependent vortex structures [16], fractional vortices that contain $\Phi_{0} / 2$ [17], and $4 \pi$ vortices $\left(2 \Phi_{0}\right)$ [18], which should all be readily detectable by our Hall probe microscope.

It is against this background of complex materials science, a desire to investigate magnetic signatures at sharp sample edges, and the predicted novel behavior in microstructures that the series of SHPM measurements described here was undertaken.

\section{EXPERIMENTAL METHOD}

Superconducting $\mathrm{Sr}_{2} \mathrm{RuO}_{4}$ single crystals were grown using the floating-zone technique with Ru self-flux in a commercial image furnace [19], and annealed in air $\left(1500{ }^{\circ} \mathrm{C}\right.$ for 3 days $)$ to remove lattice defects and reduce vortex pinning [20]. Figure 1 shows both the real $\left(\chi^{\prime}\right)$ and imaginary $\left(\chi^{\prime \prime}\right)$ components of ac susceptibility measurements (drive frequency $=71 \mathrm{~Hz}$, drive amplitude $=0.43 \mathrm{G}$ ) on the sample prior to annealing and patterning. Both curves are extremely sharp, indicative of low levels of disorder. Defining $T_{\mathrm{c}}$ as the point where $\chi^{\prime}$ falls to $10 \%$ of the low temperature value, and the transition width $\Delta T_{\mathrm{c}}$ as the full width at half maximum of $\chi^{\prime \prime}$, we find $T_{\mathrm{c}} \cong$ $1.50 \mathrm{~K}$ and $\Delta T_{\mathrm{c}} \cong 0.06 \mathrm{~K}$, which represents the current state of the art for this material, confirming that we have extremely high quality single crystals. Optical lithography and argon 


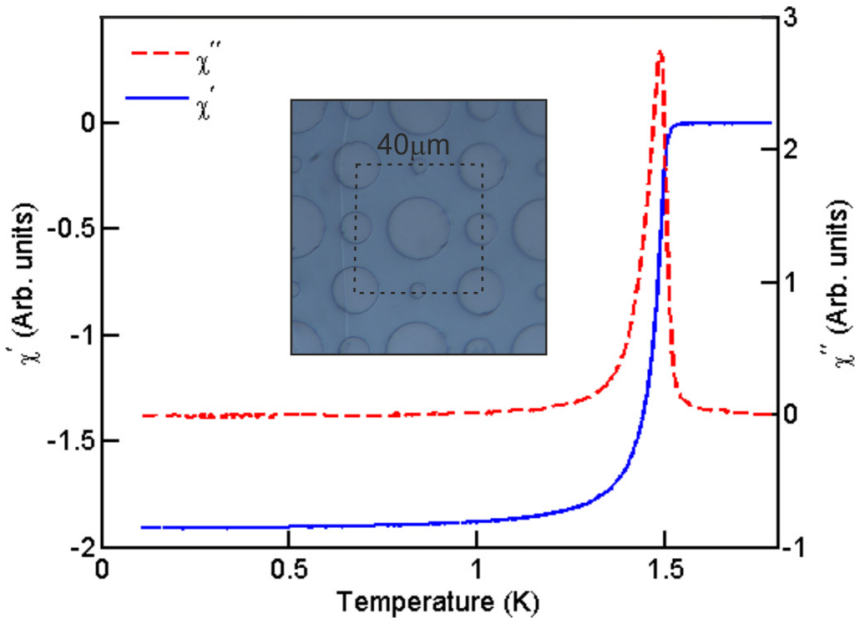

FIG. 1. (Color online) Real $\left(\chi^{\prime}\right)$ and imaginary $\left(\chi^{\prime \prime}\right)$ parts of the $\mathrm{Sr}_{2} \mathrm{RuO}_{4}$ single crystal ac susceptibility data measured through the critical temperature prior to annealing. The sharp transition $\Delta T \approx$ $0.06 \mathrm{~K}$ and high $T_{\mathrm{c}} \cong 1.50 \mathrm{~K}$ illustrate the high crystalline quality of the sample. An optical micrograph of the square array of microscopic disks etched into the $a b$ face is shown in the inset. The disks have radii of $2.5,5,7.5$, and $10 \mu \mathrm{m}$ and stand $400 \mathrm{~nm}$ proud of the surface.

ion milling were used to etch an array of shallow cylindrical pillars into the cleaved $a b$ surface to a depth of $400 \mathrm{~nm}$. The inset to Fig. 1 shows an optical micrograph of the array of well-separated disks which have radii $(R)$ of $2.5,5,7.5$, and $10 \mu \mathrm{m}$.

SHPM has been used to image the stray fields at the $a b$ surface of the sample. This employs standard scanning probe techniques to approach and scan the sample surface with a nanoscale Hall effect sensor with an integrated scanning tunneling microscope (STM) tip. The active area of the Hall cross $(800 \mathrm{~nm} \times 800 \mathrm{~nm})$ was defined in an $\mathrm{AlGaAs} / \mathrm{GaAs}$ heterostructure using electron-beam lithography and wet chemical etching. The sensor is approached towards the sample until a tunneling current is established at the STM tip. The Hall probe is then retracted a small distance (typically $\sim 100 \mathrm{~nm}$ ) from the surface to allow safe scanning at high rates. Owing to the strong surface topography of this sample, the sample-sensor separation is somewhat larger than typical and is estimated to be $\sim 1.8 \mu \mathrm{m}$ by fitting profiles of individual vortices within the disks. We note, however, that it is very challenging to uniquely identify both sample-sensor separation and $\lambda$ from such fits [21]. Further details of the microscope used for these measurements can be found elsewhere [22].

\section{RESULTS}

All of the following SHPM images were captured above the $a b$ surface with the magnetic field applied parallel to the crystalline $c$ axis. If the field is increased after cooling through $T_{\mathrm{c}}$, the sample exhibits a pronounced critical state with complete flux screening from the imaging region at low fields. Increasing the applied field to achieve full penetration leads to the entrance of multivortex bundles in an uncontrolled fashion. For this reason all of the following measurements employ a field-cooling protocol from above $T_{\mathrm{c}}$, leading to vortex distributions that are close to equilibrium.
In order to be able to distinguish spontaneous edge currents from conventional Meissner screening currents, and to reduce the probability of breaking the degeneracy of the two chiral states [18], the applied field was carefully adjusted until contributions from the Earth's field and remnant fields from the cryostat were canceled out to achieve as close to a true zero field as possible $\left(H_{\text {eff }}=0\right)$, as defined by vortex free images in the field of view. Such a sequence is displayed in Fig. 2 where the scan area was centered over a $R=5 \mu \mathrm{m}$ disk. In small fields vortices are trapped outside the disk and nucleate at preferred pinning sites close to the disk edges, where they are observed to order in an approximately triangular formation with a spacing close to that expected for an ideal triangular lattice at this field strength, $a_{\mathrm{tri}}(0.125 \mathrm{G}) \approx 13.8 \mu \mathrm{m}$. At zero effective field a ring of weak image contrast is observed near the perimeter of the disk, which was shown to be an artifact attributable to electrostatic gating of the Hall sensor. The SHPM technique requires the application of a small sample bias $(0.2 \mathrm{~V})$ to allow surface detection via tunnel currents from the grounded STM tip. The relatively high sample topography modulates the electric field between sample and sensor and creates an additional parasitic "gating" signal during scanning. A definitive test of whether image contrast is due to a real magnetic feature or a gating signal is to scan the same area above $T_{\mathrm{c}}$. Figure 2(f) contains this image and reveals that the feint disk outline observed at $H_{\text {eff }}=0$ is also present for $T>T_{\mathrm{c}}$. The numerical difference of images at the two temperatures confirms that there is no magnetic contrast in the zero field image that can be attributed to chiral edge or domain wall currents above the measurement noise threshold of $\pm 2.5 \mathrm{mG}$ (system noise level $\approx 0.7 \mathrm{mG} / \sqrt{\mathrm{Hz}}$, measurement bandwidth $13 \mathrm{~Hz}$ ). A similar analysis was conducted for the $R=2.5 \mu \mathrm{m}$ disk and returned the same result.

Figure 3(a) shows images of the same $R=5 \mu \mathrm{m}$ disk after field cooling in increasing applied fields up to 100 Oe. For low fields, the number of vortices nucleating at the disk edge increases and the disk becomes clearly visible by virtue of its complete flux screening. Eventually, at $1.25 \mathrm{Oe}$ a single vortex

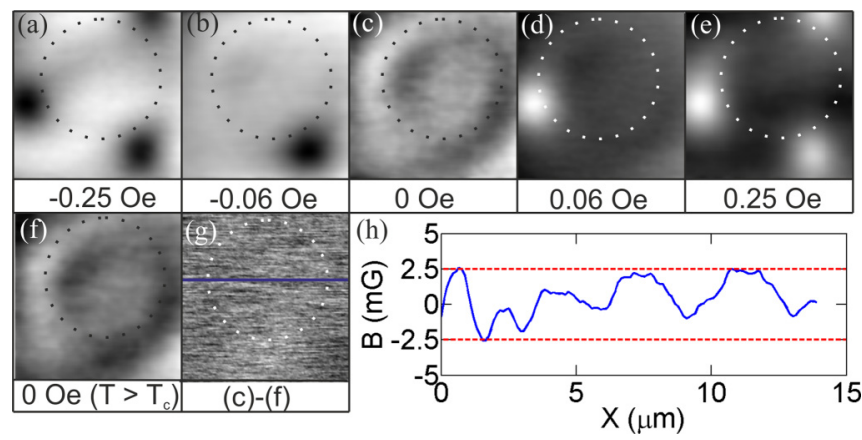

FIG. 2. (Color online) SHPM images $(14 \mu \mathrm{m} \times 14 \mu \mathrm{m})$ of a mesoscopic $\mathrm{Sr}_{2} \mathrm{RuO}_{4}$ disk (dashed line, $R=5 \mu \mathrm{m}$ ) after field cooling in small fields spanning $H_{\text {eff }}=0$ Oe. $T=260 \mathrm{mK}$. From left to right the image grayscales are $0.41,0.45,0.18,0.42,0.44,0.19$, and $0.07 \mathrm{G}$. The similarity of the images at $H_{\text {eff }}=0$, above and below $T_{\mathrm{c}}$ [(c) and (f)], indicates that any contrast in these images is entirely attributable to a gating artifact. The line scan (h) from the difference image (g) [(f) minus (c)] places an upper limit of $\pm 2.5 \mathrm{mG}$ on spontaneous fields due to chiral edge currents in these images. 


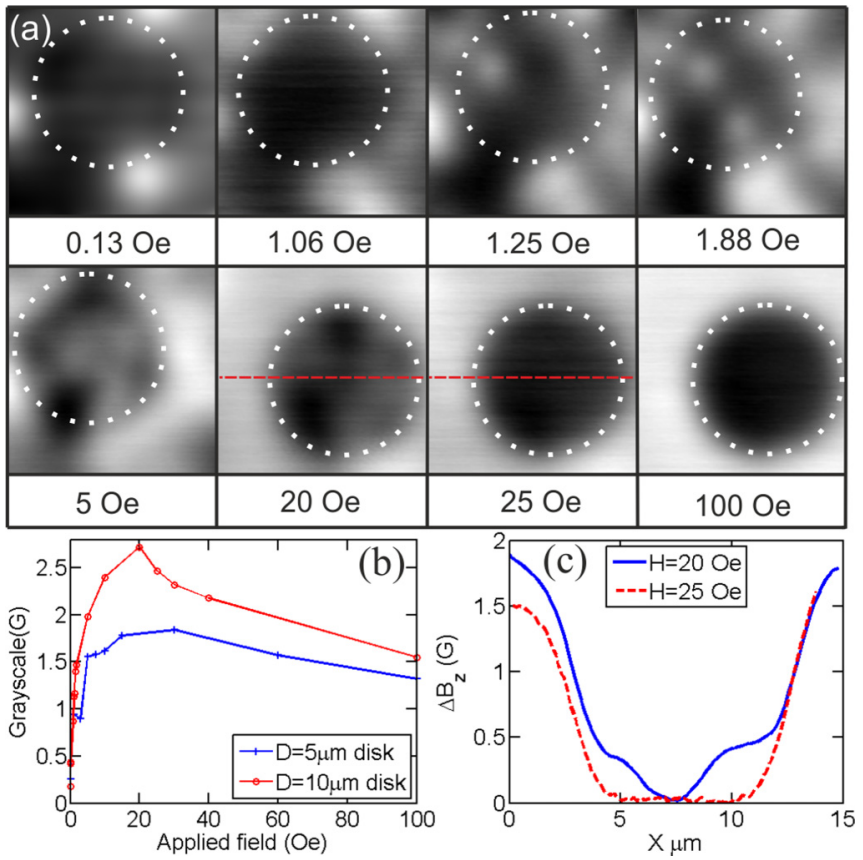

FIG. 3. (Color online) (a) A sequence of SHPM images $(14 \mu \mathrm{m} \times 14 \mu \mathrm{m})$ of a mesoscopic $\mathrm{Sr}_{2} \mathrm{RuO}_{4}$ disk $(R=5 \mu \mathrm{m})$ after field cooling in increasing magnetic fields up to $100 \mathrm{Oe}$. (b) The image grayscale which peaks at $\sim 20-25$ Oe and gradually falls with increasing field. (c) Line scans across the disks at 20 and $25 \mathrm{Oe}$, as indicated in the relevant images (a).

penetrates the disk, followed by a second at 1.88 Oe. Individual vortex resolution is lost at $\sim 5$ Oe but even in fields up to $20 \mathrm{Oe}$, when we estimate the disk already contains $\sim 70 \Phi_{0}$, a rather inhomogeneous flux distribution is resolved, suggestive of the presence of a quite strong nonuniform pinning potential in the sample. Surprisingly, at 25 Oe the flux distribution inside the disk suddenly becomes completely homogeneous within the resolution of the experiment $(\Delta B= \pm 2.5 \mathrm{mG}$, spatial resolution $\approx 1.8 \mu \mathrm{m}$ ). This is shown by the line scans across the disks at 20 and 25 Oe (dashed lines). Flux continues to preferentially enter the disk homogeneously up to the highest measurement field of $100 \mathrm{Oe}$, as demonstrated by the diminishing image grayscales shown in Fig. 3(b). These grayscales represent the difference in measured magnetic induction between regions of highest (white) and lowest (black) contrast, and therefore at these high fields (where vortices are everywhere) reflect the strength of the diamagnetic screening from the disks. Even at 100 Oe this is appreciably higher than the signal of a single isolated vortex $(\sim 0.6 \mathrm{G})$ for these measurements.

Line scans across the disk at the three highest fields, when the flux distribution has become homogeneous, reveal a steep-edged and flat-bottomed inverted "top hat" shape to the field profile (Fig. 4). Theoretical comparisons have been made with the critical state model of Clem and Sanchez [23], for microscopic disks in the high-field limit, when $J_{\mathrm{c}}$ flows everywhere within the disks [23]. The sample-sensor separation $(\sim 1.8 \mu \mathrm{m}$ during this experiment $)$ and Hall probe active width $(w=0.8 \mu \mathrm{m})$ are taken into account when simulating the experimental magnetic field profile. Figure 4
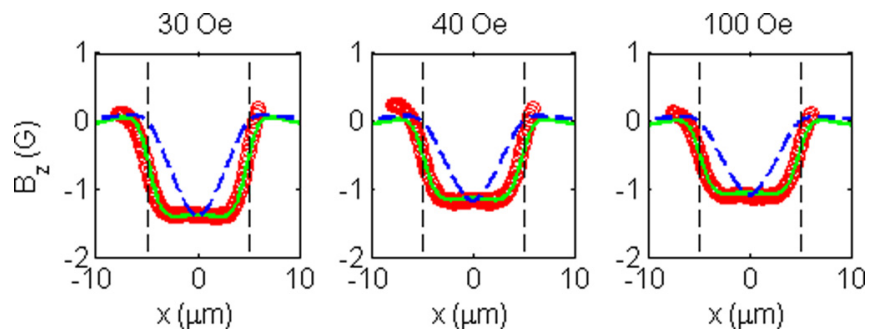

FIG. 4. (Color online) Experimental magnetic field profiles (circles) across mesoscopic $\mathrm{Sr}_{2} \mathrm{RuO}_{4}$ disks $(R=5 \mu \mathrm{m})$ captured after field cooling from $T>T_{\text {c }}$ to $T=260 \mathrm{mK}$ in applied fields of 30 , 40, and 100 Oe. Two theoretical fits are shown: first, assuming a constant bulk critical current $J_{\mathrm{c}}=7.75,6.5$, and $6.0 \times 10^{4} \mathrm{~A} \mathrm{~cm}^{-2}$, respectively (dashed lines), and a second assuming edge supercurrents $\left(J_{\mathrm{E}}=1.55,1.25\right.$, and $1.15 \times 10^{6} \mathrm{~A} \mathrm{~cm}^{-2}$, respectively) flowing within $d / 2$ of the edge of the disk and $J_{\mathrm{c}}=0$ (solid lines).

illustrates that the theoretical calculations of the disk field profile based solely on a uniform bulk current $\left(J_{\mathrm{c}}\right)$ provide a very poor fit to the observed field profiles (dashed lines). Good agreement with the experiment is only achieved when edge currents are included $\left(J_{\mathrm{E}} \gg J_{\mathrm{c}}\right)$. In practice, following the approach for the geometrical barrier [24], profiles with $J_{\mathrm{E}}$ flowing within $d / 2$ of the sample edge, where $d=400 \mathrm{~nm}$ is the disk thickness, and $J_{\mathrm{c}}=0$ are found to give excellent agreement with the experimental field profiles (solid lines, Fig. 4). In fact, the profile is dominated by $J_{\mathrm{E}}$ and rather insensitive to $J_{\mathrm{c}}$, allowing us to set a limit of $J_{\mathrm{c}}<10^{7} \mathrm{~A} \mathrm{~m}^{-2}$. For $J_{\mathrm{c}}>10^{7} \mathrm{~A} \mathrm{~m}^{-2}$ the bottom of the profile becomes more rounded and the good agreement is lost.

The theoretical fits based on edge and bulk currents are made assuming constant sample-sensor separation $(z=$ $1.8 \mu \mathrm{m}$ ) and are solely parametrized by $J_{\mathrm{E}}$ and $J_{\mathrm{c}}$, respectively. The strength of the screening of flux from the disk at high fields decreases with increasing field, as demonstrated by the decreasing magnitudes of the inverted "top hat" profiles in Fig. 4. This trend is also captured by the image grayscale values displayed in Fig. 3(b). Fits to disk profiles captured at different $H_{\text {eff }}$ therefore require a field-dependent $J_{\mathrm{E}}\left(H_{\text {eff }}\right)$, as plotted in Fig. 5.

The disk edges were observed to play a prominent role in the vortex structures formed in the $R=10 \mu \mathrm{m}$ disk at $T=$ $1 \mathrm{~K}$ shown in Fig. 6. Six vortices are seen to form a ring at the center of the disk, reflecting the rotational symmetry of the mesostructure, in what resembles a discrete analog of Zeldov's continuous flux dome [24], that is predicted to occur as a consequence of strong edge currents resulting from the geometrical barrier. The bright object at the topmost vertex of the ring contains two vortices whose separation is below the spatial resolution of our experiment. This, and the two additional vortices just inside the disk boundary, suggest that pinning forces are still playing a strong role at this temperature.

\section{DISCUSSION}

Previously attempts have been made to induce chiral currents in $\mathrm{Sr}_{2} \mathrm{RuO}_{4}$ single crystals by introducing an array of $1 \mu \mathrm{m}$ diameter, $1 \mu \mathrm{m}$ deep holes using focused ion-beam milling [25]. In practice these proved to be very weak vortex 


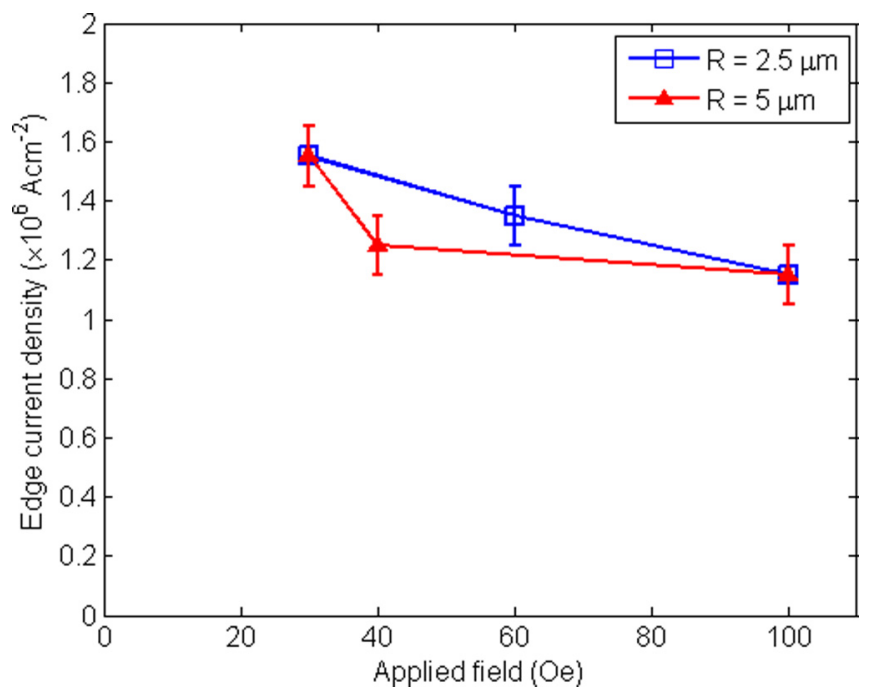

FIG. 5. (Color online) Calculated edge current densities flowing in the $R=2.5$ and $5 \mu \mathrm{m}$ disks at various applied fields.

pinning centres, and so their effectiveness as sites of broken translational symmetry generating spontaneous chiral edge currents was questioned by the authors. In contrast, the mesoscopic disks discussed here have been observed to have a profound impact on the vortex physics of the system. At very low fields (Fig. 2) vortices are screened from the disks by strong edge currents and take up locations at pinning sites on or near the disk edge, and upon penetration arrange into (disordered) rings at the disk center, presumably driven in part by currents flowing at the disk edges (Fig. 6). However, despite the presence of sharp sample edges, we see no evidence for spontaneous currents near the edge of the disks that could be attributed to a chiral order parameter, nor were we able to resolve any spontaneous currents arising at chiral domain walls. We conclude that if present, magnetic signatures from chiral edge currents and chiral domain walls are below the $\pm 2.5 \mathrm{mG}$ noise floor of this experiment.

Figure 7 presents a simulation of the magnetic field signal we would expect to measure above sample edges in a semiinfinite superconducting sample with a chiral $p$-wave order parameter, where it is assumed that the superconductor is two dimensional with a single cylindrical Fermi surface. The exact numerical solutions to the inhomogeneous London equations of Matsumoto and Sigrist [10] are complex and cumbersome

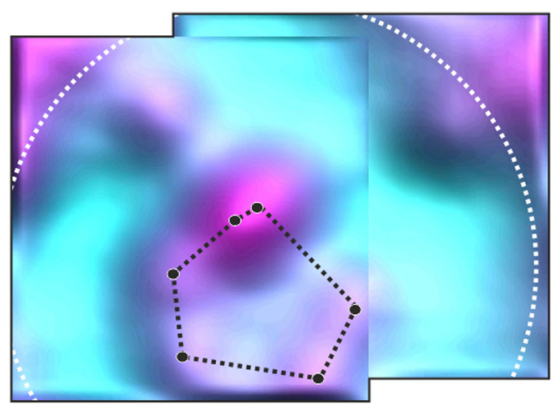

FIG. 6. (Color online) At $H_{\text {eff }}=1 \mathrm{Oe}$, six vortices form a disordered ring at the center of an $R=10 \mu \mathrm{m}$ disk at $T=1 \mathrm{~K}$. to derive. Instead, Fig. 7 presents results based on an adaption of the fitting protocol of Bluhm [Eq. (1)] [26], from which we have calculated the appropriate supercurrent density via the standard relationship [Eq. (2)], which is then used to calculate the magnetic induction distribution [Eq. (3)] using the Biot-Savart law. In addition, the plot shown in Fig. 7 includes averaging to account for the finite Hall probe active area. From this analysis we conclude that, if present, the signals at chiral edge fields are $<0.8 \%$ those predicted by this model:

$$
\begin{gathered}
B_{0, z}(x)=\frac{B_{0}}{1-\tilde{\xi}^{2} / \tilde{\lambda}^{2}}\left(e^{-|x| \mid \tilde{\lambda}}-e^{-|x| / \tilde{\xi}}\right) \\
J=\frac{1}{\mu_{0}} \nabla \times B \\
B_{\mathrm{CEF}}\left(x_{0}\right)=\frac{\frac{B_{0}}{2 \pi}}{1-\frac{\tilde{\xi}^{2}}{\tilde{\lambda}^{2}}} \\
\quad \times \int_{-\infty}^{0} \int_{0}^{\infty} \frac{\left[\frac{1}{\tilde{\lambda}} \exp \left(\frac{-x}{\tilde{\lambda}}\right)-\frac{1}{\tilde{\xi}} \exp \left(\frac{-x}{\tilde{\xi}}\right)\right]\left(x_{0}-x\right)}{\left(x_{0}-x\right)^{2}+\left(z_{0}-z\right)^{2}} \\
\quad \times d x d z .
\end{gathered}
$$

Here we have assumed $\xi=66 \mathrm{~nm}$, and adopted Bluhm's fit parameters $\tilde{\lambda}=2.2 \xi$ and $\tilde{\xi}=1.5 \xi . B_{0}$ is an additional fitting parameter introduced by Bluhm, which he took to be $87 \mathrm{G}$ in order to match the field scale of the numerical calculations.

Earlier attempts using scanning SQUID microscopy were made to resolve chiral currents (fields) at the less-well-defined as-grown edges of $\mathrm{Sr}_{2} \mathrm{RuO}_{4}$ single crystals with a slightly lower $T_{\mathrm{C}}$ than those used here $[13,15]$. These authors also failed to find any evidence for them and put a conservative limit on chiral edge field signals in their samples at $<3 \%$ of theoretical expectations. The measurements presented here therefore reduce this upper bound by a factor of $\sim 4$, in an imaging system with a superior spatial resolution (by a factor of $\sim 2$ ), on well-defined microstructures patterned in state-ofthe-art annealed single crystals $\left(T_{\mathrm{c}} \cong 1.50 \mathrm{~K}\right)$. Given the ever tightening constraints on the magnitude of chiral currents in $\mathrm{Sr}_{2} \mathrm{RuO}_{4}$, there is an increased focus on explanations for their apparent absence ranging from disorder, band anisotropy and surface scattering effects [27], complete retroflection at the surface [28], and even attempts to move beyond the BCS or Bogliubov-de Gennes formalism [29]. All of these are well discussed in a recent review [2].

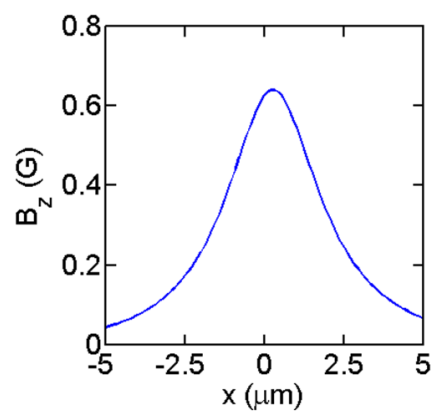

FIG. 7. (Color online) Simulation of an SHPM measurement of stray fields above a semi-infinite chiral $p$-wave superconductor $(x>$ 0 ) at a height of $1.8 \mu \mathrm{m}$ due to chiral edge currents. 
Figure 3(a) shows the field distribution in a $R=5 \mu \mathrm{m}$ disk after field cooling from $T>T_{\mathrm{c}}$ in applied fields up to $100 \mathrm{Oe}$. At low fields the first vortex enters the disk at 1.25 Oe and similar images allow us to track this penetration field as a function of disk radius. Within our limited data set (four disk radii) the behavior is in approximate agreement with established theories of surface barriers [24,30,31], and recent investigations of vortex penetration and expulsion in field-cooled $\mathrm{YB}_{2} \mathrm{Cu}_{3} \mathrm{O}_{7-\delta}$ and $\mathrm{Nb}$ strips [32].

As the field is increased further, the vortex density in the disk increases and single vortex resolution is lost. Previous studies of similar disks in $\mathrm{Bi}_{2} \mathrm{Sr}_{2} \mathrm{CaCu}_{2} \mathrm{O}_{8+\delta}$ (BSCCO-2212) have captured evidence of a vortex "dome" forming at the center of the disk, consistent with the theory of the geometrical barrier [33]. In $\mathrm{Sr}_{2} \mathrm{RuO}_{4}$ at $300 \mathrm{mK}$, in applied fields up to 20 Oe, we do not observe a smooth dome but instead a rather inhomogeneous vortex distribution is captured, indicative of a low density of rather strong pinning sites. Tellingly, the grayscale at $20 \mathrm{Oe}$ of $2.72 \mathrm{G}$ is about four times greater than that for a single isolated vortex $(\sim 0.6 \mathrm{G})$, reflecting the fact that the vortices are screened by the strong edge currents resulting in high contrast between the disk and the interstitial regions.

It is therefore surprising that at 25 Oe the vortex distribution inside the disk abruptly becomes homogeneous. It is as if the pinning forces responsible for the inhomogeneity are suddenly "turned off" at this field strength. Interestingly, this also coincides with a peak in the field profile across the disk, which falls quite steeply at higher fields $\left(H_{\mathrm{eff}} \geqslant 25 \mathrm{Oe}\right)$, as captured by the gradually decreasing grayscales of the images [Fig. 3(b)]. Previous theoretical work has predicted that a rotation of the order-parameter $d$ vector into the $a-b$ plane can occur at sufficiently high $c$-axis fields, resulting in a profound change of behavior of the system $[34,35]$. In this instance the Cooper pair spins would be parallel to the external field and the degeneracy of the two chiral states would be lifted. More recently, four component Ginzburg-Landau models predicted that three different order parameters can be stabilized in the $H-T$ phase diagram for fields applied along the $c$ axis [36]. The exact field at which the rotation occurs is not well known but an upper bound of 200 Oe has been suggested [34]. The abrupt change in vortex pinning behavior above 20-25 Oe may be the signature of a field-driven change of the order parameter of this type. We also note that the abrupt change in pinning behavior occurs close to the maximum field at which a dramatic increase in the Meissner fraction was recently observed in $\mu \mathrm{SR}$ measurements at low temperatures, something that was tentatively attributed to a long-range vortex attraction arising from multiband effects [37]. Hence it is possible that these two observations have a common physical origin.

Line scans of the disks at $H_{\text {eff }} \geqslant 25$ Oe display a steepsided and flat-bottomed magnetic profile (Fig. 4). Critical state theory for saturated thin disks [23] yields very poor agreement with experiment (cf. the dashed lines in Fig. 4). However, good agreement is reached with a field profile derived from a model containing only edge currents $\left(J_{\mathrm{E}}\right)$ flowing within a distance $d / 2$ of the sample edge (cf. the solid lines in Fig. 4).

The edge currents used in the disk profile fitting shown in Fig. 4 are $J_{\mathrm{E}} \sim 2 \times 10^{10} \mathrm{~A} \mathrm{~m}^{-2}$ and compare favorably with theoretical predictions of the geometrical barrier, $J_{\mathrm{E}}^{\mathrm{GB}}=$ $2 H_{\mathrm{c} 1} / d \sim 10^{10} \mathrm{~A} \mathrm{~m}^{-2}$ [24] $\left(H_{\mathrm{c} 1} \approx 70\right.$ Oe [38]), providing confidence that the geometrical barrier model is appropriate for our sample. Note that these are two orders of magnitude smaller than the theoretical depairing current, $J_{\mathrm{dp}} \approx$ $B_{\mathrm{c}} / 2 \mu_{0} \lambda \sim 10^{12} \mathrm{~A} \mathrm{~m}^{-2}$. The edge current fitting procedure was also able to put an upper bound on $J_{\mathrm{c}}<10^{7} \mathrm{~A} \mathrm{~m}^{-2}$.

In order to accurately replicate the magnetic profiles of the disks the fitting required $J_{\mathrm{E}} \gg J_{\mathrm{c}}$, which corresponds to the weak pinning regime [24]. This is seemingly in direct conflict with the indications of strong pinning observed below $25 \mathrm{Oe}$ in this experiment, and in previous SHPM measurements of the same sample [14]. Up to now we have ignored the fact that the "disks" of this study are not free standing but are on top of $\mathrm{a} \sim 1 \mathrm{~mm}$ thick $\mathrm{Sr}_{2} \mathrm{RuO}_{4}$ platelet. If bulk pinning were strong, one would expect this to dominate over the role of the relatively thin microfabricated disk. However, in the limit $J_{\mathrm{c}}=0$ it can be completely ignored. The fact that the disk plays such a dominant role at all fields is also consistent with a small value of $J_{\mathrm{c}}$.

The disks studied here are in the regime $R \ll \xi_{0}$ and $R \sim 25 \lambda$ and so are only nominally in the mesoscopic limit, except very close to $T_{\mathrm{c}}$ [39]. It is therefore interesting that we observe the formation of a (disordered) vortex ring, reflecting the rotational disk symmetry, at the center of the $R=10 \mu \mathrm{m}$ disk at $T=1 \mathrm{~K}\left(H_{\mathrm{eff}}=1 \mathrm{Oe}\right)$. This provides direct evidence of the influence of the disk boundaries on the internal vortex configuration. Many images at low temperature have provided evidence of a low density of strong pinning sites in this sample. In this instance the formation of a vortex ring is probably aided by the increased measurement temperature of $1 \mathrm{~K}$ which facilitates the thermal excitation of vortices off the pinning sites, allowing the intrinsic vortex-vortex interactions to determine the vortex configuration. The formation of such a vortex ring is consistent with predictions for "large" mesoscopic disks [40].

\section{CONCLUSION}

Large mesoscopic disks have been milled into the $a b$ surface of a high quality $\mathrm{Sr}_{2} \mathrm{RuO}_{4}$ single crystal in order to provide well-defined regions of broken translational symmetry where current theory for the $\hat{d}=\Delta_{0}\left(k_{x} \pm i k_{y}\right) \hat{z}$ chiral order parameter predicts spontaneous chiral currents (fields) should form. Scanning Hall probe images reveal no spontaneous magnetic signal at the disk edges above the experimental noise threshold of $\pm 2.5 \mathrm{mG}$, placing an upper limit of $\approx 0.8 \%$ of theoretical predictions. Neither do we observe any magnetic signal anywhere else in the sample that could be attributed to the signature of a chiral domain wall. After field cooling from $T>T_{\mathrm{c}}$ the disks strongly screen magnetic flux at the highest fields. Theoretical fits to magnetic field profiles of the disks for $H_{\text {eff }}>25$ Oe indicate that the screening is dominated by edge currents flowing within $d / 2$ of the disk edge, and that the system is in the weak pinning limit $\left(J_{\mathrm{E}} \gg J_{\mathrm{c}}\right)$. This final observation is in direct contradiction with several pieces of evidence that suggest the presence of a low density of rather strong pinning sites at low temperatures and low fields $\left(H_{\text {eff }}<25 \mathrm{Oe}\right)$. An abrupt change in the vortex pinning and screening behavior above 25 Oe may indicate a field-driven change in the order parameter. 


\section{ACKNOWLEDGMENTS}

We would like to acknowledge extremely valuable and insightful discussions with the late John Clem and stimulating discussion with James Annett. This work was supported by the Engineering and Physical Sciences Research Council (EPSRC) in the United Kingdom under Grant No. EP/J010626/1.
[1] M. R. Norman, arXiv:1302.3176.

[2] C. Kallin, Rep. Prog. Phys. 75, 042501 (2012).

[3] Y. Maeno, S. Kittaka, T. Nomura, S. Yonezawa, and K. Ishida, J. Phys. Soc. Jpn. 81, 011009 (2012).

[4] K. Ishida, H. Mukuda, Y. Kitaoka, K. Asayama, Z. Q. Mao, Y. Mori, and Y. Maeno, Nature (London) 396, 658 (1998).

[5] M. Sigrist and K. Ueda, Rev. Mod. Phys. 63, 239 (1991).

[6] G. M. Luke, Y. Fudamoto, K. M. Kojima, M. I. Larkin, J. Merrin, B. Nachumi, Y. J. Uemura, Y. Maeno, Z. Q. Mao, Y. Mori, H. Nakamura, and M. Sigrist, Nature (London) 394, 558 (1998).

[7] J. Xia, Y. Maeno, P. T. Beyersdorf, M. M. Fejer, and A. Kapitulnik, Phys. Rev. Lett. 97, 167002 (2006).

[8] Y. Maeno, H. Hashimoto, K. Yoshida, S. Nishizaki, T. Fujita, J. G. Bednorz, and F. Lichtenberg, Nature (London) 372, 532 (1994).

[9] A. P. Mackenzie and Y. Maeno, Rev. Mod. Phys. 75, 657 (2003).

[10] M. Matsumoto and M. Sigrist, J. Phys. Soc. Jpn. 68, 994 (1999).

[11] M. Stone and R. Roy, Phys. Rev. B 69, 184511 (2004).

[12] V. O. Dolocan, C. Veauvy, F. Servant, P. Lejay, K. Hasselbach, Y. Liu, and D. Mailly, Phys. Rev. Lett. 95, 097004 (2005).

[13] C. W. Hicks, J. R. Kirtley, T. M. Lippman, N. C. Koshnick, M. E. Huber, Y. Maeno, W. M. Yuhasz, M. B. Maple, and K. A. Moler, Phys. Rev. B 81, 214501 (2010).

[14] P. J. Curran, V. V. Khotkevych, S. J. Bending, A. S. Gibbs, S. L. Lee, and A. P. Mackenzie, Phys. Rev. B 84, 104507 (2011).

[15] J. R. Kirtley, C. Kallin, C. W. Hicks, E. A. Kim, Y. Liu, K. A. Moler, Y. Maeno, and K. D. Nelson, Phys. Rev. B 76, 014526 (2007).

[16] J.-W. Huo, W.-Q. Chen, S. Raghu, and F.-C. Zhang, Phys. Rev. Lett. 108, 257002 (2012).

[17] S. B. Chung, H. Bluhm, and E.-A. Kim, Phys. Rev. Lett. 99, 197002 (2007).

[18] M. Ichioka, Y. Matsunaga, and K. Machida, Phys. Rev. B 71, 172510 (2005).

[19] Y. Maeno, S. Nishizaki, K. Yoshida, S. Ikeda, and T. Fujita, J. Low Temp. Phys. 105, 1577 (1996).

[20] Z. Q. Mao, Y. Maeno, and H. Fukazawa, Mater. Res. Bull. 35, 1813 (2000).

[21] V. G. Kogan, Phys. Rev. B 68, 104511 (2003).
[22] V. V. Khotkevych, M. V. Milosevic, and S. J. Bending, Rev. Sci. Instrum. 79, 123708 (2008).

[23] J. R. Clem and A. Sanchez, Phys. Rev. B 50, 9355 (1994).

[24] E. Zeldov, A. I. Larkin, V. B. Geshkenbein, M. Konczykowski, D. Majer, B. Khaykovich, V. M. Vinokur, and H. Shtrikman, Phys. Rev. Lett. 73, 1428 (1994).

[25] P. G. Bjornsson, Y. Maeno, M. E. Huber, and K. A. Moler, Phys. Rev. B 72, 012504 (2005).

[26] H. Bluhm, Phys. Rev. B 76, 144507 (2007).

[27] P. E. C. Ashby and C. Kallin, Phys. Rev. B 79, 224509 (2009).

[28] J. A. Sauls, Phys. Rev. B 84, 214509 (2011).

[29] A. Leggett, Quantum Liquids: Bose Condensation and Cooper Pairing in Condensed-Matter Systems (Oxford University Press, Oxford, U.K., 2006).

[30] L. Burlachkov, V. B. Geshkenbein, A. E. Koshelev, A. I. Larkin, and V. M. Vinokur, Phys. Rev. B 50, 16770 (1994).

[31] G. Blatter, M. V. Feigelman, V. B. Geshkenbein, A. I. Larkin, and V. M. Vinokur, Rev. Mod. Phys. 66, 1125 (1994).

[32] K. H. Kuit, J. R. Kirtley, W. van der Veur, C. G. Molenaar, F. J. G. Roesthuis, A. G. P. Troeman, J. R. Clem, H. Hilgenkamp, H. Rogalla, and J. Flokstra, Phys. Rev. B 77, 134504 (2008).

[33] M. R. Connolly, M. V. Milosevic, S. J. Bending, J. R. Clem, and T. Tamegai, Europhys. Lett. 85, 17008 (2009).

[34] J. F. Annett, B. L. Gyorffy, G. Litak, and K. I. Wysokinski, Phys. Rev. B 78, 054511 (2008).

[35] H. Murakawa, K. Ishida, K. Kitagawa, Z. Q. Mao, and Y. Maeno, Phys. Rev. Lett. 93, 167004 (2004).

[36] S. Takamatsu and Y. Yanase, J. Phys. Soc. Jpn. 82, 063706 (2013).

[37] S. J. Ray, A. S. Gibbs, S. J. Bending, P. J. Curran, E. Babaev, C. Baines, A. P. Mackenzie, and S. L. Lee, Phys. Rev. B 89, 094504 (2014).

[38] K. Deguchi, Z. Mao, and Y. Maeno, J. Phys. Soc. Jpn. 73, 1313 (2004).

[39] V. A. Schweigert, F. M. Peeters, and P. S. Deo, Phys. Rev. Lett. 81, 2783 (1998).

[40] V. R. Misko, B. Xu, and F. M. Peeters, Phys. Rev. B 76, 024516 (2007). 